\title{
Interprofissionalidade na atenção primária: intencionalidades das equipes versus realidade do processo de trabalho
}

\author{
Interprofessional collaboration in primary health care: the team's intentions versus the reality of work \\ processes
}

La colaboración interprofesional en la atención primaria: la intencionalidad de los equipos versus la realidad del proceso de trabajo

\author{
Aridiane Alves Ribeiro ${ }^{1}$ (1) \\ Christiane Ricaldoni Giviziez ${ }^{1}$ (1) \\ Elânia Assis Rocha Coimbra² (1) \\ Jeniffer Dayane Duarte dos Santos ${ }^{1} \mathbb{C}$ \\ Jhonatan Emanuel Maciel de Pontes ${ }^{1}$ (1) \\ Nina Franco Luz ${ }^{1}$ (1) \\ Rafael de Oliveira Rocha ${ }^{1}$ (D)
}

Webster Leonardo Guimarães da Costa $^{1}$ (1)

1. Universidade Federal de Jataí. Jataí, GO, Brasil.

2. Secretaria Municipal de Saúde de Jataí. Jataí, GO, Brasil.
Autor correspondente:

Aridiane Alves Ribeiro.

E-mail: aridianeribeiro@gmail.com

Recebido em 01/06/2021.

Aprovado em 11/08/2021.

DOI:https://doi.org/10.1590/2177-9465-EAN-2021-0141

\section{Resumo}

Objetivo: comparar atitudes relacionadas à colaboração interprofissional autorrelatadas por diferentes equipes da atenção primária com a realidade observada de seus processos de trabalho. Método: abordagem qualitativa e quantitativa implementada em duas etapas de coleta de dados, entre dezembro de 2019 e outubro de 2020. Na qualitativa, empregou-se a observação sistemática dos atendimentos em unidades de saúde. Utilizou-se roteiro de observação baseado no Referencial para Competências em Interprofissionalidade e no Fluxograma Analisador do processo de trabalho centrado no usuário. As observações foram registradas em diário de campo. Na etapa quantitativa, aplicou-se a Escala de Atitudes Relacionadas à Colaboração Interprofissional. Resultados noventa e um profissionais da atenção básica responderam a escala. Obteve-se uma média de 120 de pontuação, que significa valorização do trabalho colaborativo por respondentes de todas as equipes. Apesar disso, foram observadas divergências entre o falado e o vivido, pois o processo de trabalho estruturado parece limitar atitudes colaborativas. Conclusões e implicações para a prática: valorização de atividades programadas para o trabalho interprofissional da equipe de saúde e de enfermagem na atenção primária, como espaços na agenda para reuniões. Necessidade de promoção da educação interprofissional com trabalhadores, bem como de políticas públicas que garantam mecanismos para o trabalho colaborativo na atenção básica.

Palavras-chave: Ambiente de Trabalho Colaborativo; Atenção Primária à Saúde; Fluxo de trabalho; Equipe de trabalho; Relações Interprofissionais.

\begin{abstract}
Objective: to compare self-reported interprofessional attitudes according to the teams from different primary health care services with the actual context of work processes. Method: Quantitative and qualitative study in which data were collected in two stages between December of 2019 and October of 2020. Systematic observation was the strategy used to collect data from primary health care centers. A script based on the Canadian Interprofessional Health Collaborative framework and the Analyzer Flowchart was used to observe the patient-centered work process. Observations were recorded in a Field Diary, and the Brazilian version of the Jefferson Scale of Attitudes Toward Interprofessional Collaboration was used. Results: 91 primary health workers completed the scale. The median score was 120 , meaning all the health teams value collaborative work. Nonetheless, differences were found between what the participants say and what they do, considering that the structured work process limits collaborative practice. Conclusions and Implications to Practice: the services need to value programmed activities to promote interprofessional collaboration among health and nursing teams working in primary health care, and reserve time, and give opportunities for meetings to occur. Additionally, training addressing interprofessional collaboration needs to be provided to workers in addition to public policies to ensure mechanisms that promote collaborative practices.
\end{abstract}

Keywords: Working Environment; Primary Health Care; Workflow. Workforce; Interprofessional Relations.

\section{Resumen}

Objetivo: comparar las actitudes relacionadas con la colaboración interprofesional autoinformada por diferentes equipos de la atención primaria con la realidad observada en sus procesos de trabajo. Método: investigación cualitativa y cuantitativa. La recolección de datos ocurrió entre diciembre de 2019 y octubre de 2020. En la etapa cualitativa se utilizó la observación sistemática de la atención en las unidades de salud, con un guión de observación basado en el Referencial de Competencias Interprofesionales y el Diagrama Analizador del Flujo del proceso de trabajo centrado en el usuario. Registramos las observaciones en el diario de campo. En la etapa cuantitativa se aplicó la Escala de Actitudes Relacionadas con la Colaboración Interprofesional. Resultados: 91 profesionales respondieron la escala. Se obtuvo una media de 120 puntos, lo que supone valorización del trabajo colaborativo por profesionales de todos los equipos evaluados. Sin embargo, verificamos diferencias entre lo relatado y lo vivido, ya que el proceso de trabajo estructurado parece limitar las actitudes para el trabajo colaborativo. Conclusiones e implicaciones para la práctica: valorización de actividades programadas para el trabajo interprofesional de los equipos de salud y de enfermería, como reuniones programadas. Necesidad de promoción de la educación interprofesional, así como de políticas públicas que garanticen mecanismos de trabajo colaborativo.

Palabras clave: Ambiente de Trabajo; Atención Primaria de la Salud; Flujo de trabajo; Recursos Humanos; Relaciones Interprofesionales. 


\section{INTRODUÇÃO}

Diante das necessidades de saúde cada vez mais complexas, a resolutividade dos serviços perpassa a abordagem integral do indivíduo composta por diversos saberes. Em uma direção contrária, dificuldades significativas expressas por uma rígida divisão do trabalho, marcada pela fragmentação profissional, ocorrem cotidianamente nos três níveis de atenção à saúde do Sistema Único de Saúde (SUS) ${ }^{1-2}$. Nesse panorama, a interprofissionalidade vem se caracterizando como caminho para viabilizar a organização da atenção em saúde em uma perspectiva de práticas clínicas ampliadas centralizadas no indivíduo, família e comunidade ${ }^{3}$.

A interprofissionalidade ocorre quando profissionais de diferentes formações desenvolvem o trabalho em equipe, cuja colaboração perpassa planejamento e implementação das ações em saúde ${ }^{4}$. Para tanto, os trabalhadores devem atuar de modo compatível com o mesmo propósito clínico a partir de relações solidárias recíprocas, além da busca por práticas participativas com os usuários envolvidos ${ }^{5}$.

A atenção primária à saúde (APS) tem papel central na reorganização dos serviços de saúde previstos no SUS. Desde 1996, a APS é concretizada no país por meio da implementação da estratégia de saúde da família, que objetiva promover um modelo de APS pautado na coordenação do cuidado e respeitando os princípios da integralidade, universalidade e equidade previstos no SUS, tendo o trabalho em equipe como uma de suas diretrizes operacionais ${ }^{1,3}$.

Dessa forma, interprofissionalidade é um modo de agir imprescindível para se atingir a integralidade do cuidado na APS $^{6}$. De acordo com a nova Política Nacional da Atenção Básica $(\mathrm{PNAB})^{7}$, compete a todos os profissionais integrar os diferentes saberes, áreas técnicas e níveis de atenção, de acordo com as necessidades e demandas de saúde da população?.

A última versão $\mathrm{PNAB}^{7}$ apresenta mudanças, com destaque para as equipes. Prevê a manutenção, dentre outras, da equipe de Saúde da Família (eSF), da equipe de Saúde Bucal (eSB) e da equipe do Núcleo Ampliado de Saúde da Família e Atenção Primária (eNASF). E criou uma modalidade, a equipe da Atenção Básica $(e A B)^{7}$. Essa alteração foi amplamente criticada por entidades e teóricos, por significar um retrocesso dos avanços na implementação do SUS para uma época em que a APS era seletiva, focalizada e reduzida ${ }^{2,8}$.

Desde então, outras decisões políticas impactaram a APS no Brasil, vide o Programa Saúde na Hora, cuja recomendação altera o horário de atendimento nas UBS. E a nova forma de financiamento da atenção básica, que altera o indicador de captação de recursos pelos municípios para número de pessoas cadastradas².

Todas essas mudanças normativas alteram a micropolítica nas unidades básicas de saúde (UBS), especialmente o ambiente colaborativo de trabalho ${ }^{9}$, podendo ocasionar um descompasso entre as normas institucionais e prática profissional ${ }^{8}$, entre o "falado" e o "vivido"10. A implementação da interprofissionalidade na realidade brasileira é recente e desafiadora ${ }^{4}$, aspecto, potencialmente, afetado pelas mudanças no processo de trabalho e nas equipes.

Mediante essa conjuntura, questiona-se: como é o trabalho (colaborativo) nesse cenário que demanda um agir interdisciplinare entre equipes? Há diferenças em relação à interprofissionalidade no cuidado prestado entre as equipes da APS? Há divergências entre o falado e o vivido no trabalho em equipe? A partir dessas questões, este estudo objetivou comparar as atitudes relacionadas à colaboração interprofissional autorrelatadas por diferentes equipes da atenção primária coma realidade observada de seus processos de trabalho.

\section{MÉTODOS}

Este estudo é de abordagem quantitativa-qualitativa, transversal e exploratória ${ }^{11}$. O cenário do estudo foi composto por diferentes serviços da atenção primária à saúde (APS) do município referência de uma regional de saúde, localizada no interior de Goiás, composta por dez cidades, cujas populações perfazem um total de 215.282 pessoas $^{12}$.

O município sede da regional, local deste estudo, possui população de 100.882 habitantes, na qual 80.730 (80.02\%) possuem cobertura da APS, composta por 11 unidades básicas de saúde, sendo dez urbanas e uma rural, onde uma equipe de eAB e 21 equipes eSF prestam atendimento ${ }^{12-13}$.

As duas etapas da pesquisa, qualitativa e quantitativa, foram realizadas no período de dezembro de 2019 a outubro de 2020. Desenvolvidas, separadamente, a fim de evitar possível viés relacionado à interferência das diferentes técnicas utilizadas em cada uma das etapas, que seguem detalhadas a seguir. É importante comentar que a etapa qualitativa ocorreu antes do início da pandemia do Covid-19. E a fase quantitativa foi realizada durante a pandemia.

\section{Etapa qualitativa}

Na etapa qualitativa, foi utilizada a observação como técnica de coleta de dados. A seleção das UBS para essa etapa seguiu critérios, a fim de garantir representatividade e compreensão geral dos processos de trabalho das unidades componentes da rede de saúde estudada. Adotou-se o seguinte: i) UBS com eAB ou UBS com eSF e eSB; ii) localização em área urbana.

Foram incluídos neste estudo seis serviços: uma UBS com eAB e cinco com eSF e eSB. Dessas, três possuem localização centralizada e três são periféricas e todas possuem áreas de abrangência extensas. A equipe do Núcleo Ampliado de Saúde da Família e da Atenção Básica (eNASF) não foi incluída na fase qualitativa, pois atende um número limitado de unidades no município.

Foi realizada observação do tipo sistemática ${ }^{11}$ dos atendimentos realizados nos serviços de saúde selecionados por um período de dois meses. Foram observadas as relações e interações entre os profissionais e com os usuários, bem como aspectos referentes ao acolhimento, triagem, consulta individual, atendimento coletivo, reunião em equipe e referência/ contrarreferência. As observações e impressões foram registradas no Diário de Campo ${ }^{11}$.

Para tanto, foram utilizados dois instrumentos: roteiro elaborado pela equipe de pesquisa baseado no Referencial para Competências em Interprofissionalidade, do Instituto de 
Colaboração de Interprofissionalidade em Saúde do Canadá ${ }^{14} \mathrm{e}$ o Fluxograma Analisador 9 .

O Referencial para Competências em Interprofissionalidade apresenta seis domínios de competências essenciais para a prática colaborativa, sendo eles: clareza das funções, cada trabalhador compreende as atribuições específicas de cada categoria profissional; cuidado centrado no usuário, família e comunidade, todos envolvidos são integrados e engajados na implementação da assistência; funcionamento da equipe, os profissionais entendem os princípios da dinâmica do trabalho em equipe de modo a permitir uma efetiva colaboração; liderança colaborativa, trabalhadores compartilham as decisões, podendo a liderança ser exercida natural e livremente por todos; comunicação interprofissional, todos se comunicam de forma colaborativa, responsiva e responsável; e resolução de conflito interprofissional, trabalhadores se engajam e os envolvidos, incluindo usuários e família, resolução de possíveis tensões durante o processo de cuidado, reconhecendo a natureza positiva desses desentendimentos ${ }^{14}$.

O Fluxograma Analisador ${ }^{9}$ prevê o uso de figuras geométricas para expressar o atendimento centrado nos usuários produzido no processo de trabalho em saúde. As figuras são: elipse para identificar momentos de entrada e saída do usuário do serviço; losango para descrever momentos decisórios no fluxo de atendimento; $\mathrm{e}$ quadrado para caracterizar o cardápio de intervenções prestadas. ${ }^{9}$

A fim de expressar a interprofissionalidade no Fluxograma Analisador ${ }^{9}$, acrescentamos dois símbolos: ponto de exclamação e ziguezague. A exclamação refere situações de trabalho colaborativo. O ziguezague sinaliza ausências de comunicação e colaboração naquela etapa do atendimento. As equipes das seis UBS foram abordadas concomitantemente para etapa qualitativa. Os pesquisadores solicitaram autorização e permissão para acompanhar a rotina dos serviços.

Procedeu-se a análise de conteúdo temática ${ }^{15}$ dos registros do diário de campo com uso do software NVivo. O material foi transcrito para o programa, no qual foi analisado seguindo os passos: i) identificação do material conforme as UBS selecionadas; ii) leitura flutuante da totalidade do material; iii) codificação do material; iv) exploração dos dados codificados de modo a identificar diferenças e similaridades na organização dos serviços; v) categorização conforme o Fluxograma Analisador ${ }^{9}$ com diferenciação entre as UBS com eSF e UBS com eAB; vi) inferência ${ }^{15}$. A partir das categorias temáticas construídas, o Fluxograma Analisador ${ }^{9}$ foi elaborado no software Corel Draw 2020.

\section{Etapa quantitativa}

Após as observações, os profissionais foram convidados a participarem da etapa quantitativa. Enfermeiros e técnicos de enfermagem, médicos, agentes comunitários de saúde (ACS), dentistas, auxiliares de saúde bucal e outras categorias profissionais atuantes no eNASF fizeram parte dessa etapa, por amostragem de conveniência. Não foram incluídos profissionais de férias ou de licença por motivos de doença.

A Escala Jefferson de Atitudes Relacionadas à Colaboração Interprofissional (EJARCI) foi utilizada. Sua versão original ${ }^{16}$ foi traduzida e validada, em 2015, para a realidade brasileira ${ }^{17}$ É composta por 20 itens, a serem respondidos mediante uma escala do tipo Likert, de concordância/discordância, variando entre um e sete. Discordo completamente (1) corresponde ao menor nível, concordo completamente (7) se refere ao maior ${ }^{16}$

Questões sociodemográficas também foram utilizadas, a fim de obter informações sobre sexo, idade, categoria profissional, histórico de formação e atuação profissional. O instrumento sociodemográfico e a EJARCI foram enviados conjuntamente via Google Forms, por meio de rede social (WhatsApp) e e-mail, para os trabalhadores de todos os serviços de APS, incluindo as UBS observadas. Os contatos dos profissionais foram obtidos junto à Secretaria Municipal de Saúde.

A estatística descritiva foi realizada pelo software Statistical Package for the Social Sciences (SPSS) versão 24.0. As variáveis contínuas foram representadas por média \pm desvio padrão. As pontuações da EJARCI foram feitas com base nas pontuações brutas (média e desvio-padrão) e com base nos itens isolados (mediana e moda). Os dados categóricos foram apresentados em frequência absoluta e percentual (\%).

A pesquisa recebeu aprovação pelo Comitê de Ética em Pesquisa (CEP) com seres humanos da universidade vinculada ao estudo. Devido à pandemia do novo coronavírus, o estudo possui aprovação inicial sob Parecer no 3707497 de 14/11/2019e Parecer $\mathrm{n}$-4.144.874 de 09/07/2020, referente à emenda ao CEP de solicitação de ajustes na coleta de dados face à pandemia.

Os respondentes das entrevistas estruturadas concordaram em participar da pesquisa mediante leitura do Termo de Consentimento Livre e Esclarecido (TCLE). Após a anuência registrada, receberam uma cópia em seus respectivos e-mails. A equipe salvou outra cópia do termo com anuência em formato digital no computador da universidade.

\section{RESULTADOS}

\section{Caracterização dos participantes e dos serviços de atenção primária}

Participaram da pesquisa 91 respondentes do instrumento EJARCI. Trinta e cinco recusaram o convite ou não responderam as três tentativas de contato da equipe. Atribuímos essa alta taxa de recusa ao período da pandemia, com estes profissionais na linha de frente de uma pandemia.

Dentre os participantes, 91,2\% eram mulheres. A média de idade foi de 40,81 ( $\pm 10,02)$ anos. A média do tempo de formação foi de $11,21( \pm 8,42)$ anos, sendo que $56 \%$ apresentavam ensino superior. A média do tempo de atuação no SUS foi de 10,94 $( \pm 7,65)$ anos e na APS de 7,42 $( \pm 6,21)$ anos. Na Tabela 1, está apresentada a média de pontuações da EJARCI por categorias profissionais, subdivididas em dois grupos: i) eAB, eSF, eSB; e ii) eNASF.

Entre os profissionais respondentes, 69 (75,8\%) eram da eSF e $8(8,8 \%)$ da eAB. A média geral de pontuação da EJARCI foi $120( \pm 10,92)$ sendo $119( \pm 10,63)$ para as equipes de saúde da família e de saúde bucal e $126( \pm 6,93)$ para a eNASF. Ou seja, todas as categorias profissionais, independente do local de 
Tabela 1 - Média de pontuações da EJARCI por categorias profissionais subdivididas em dois grupos de equipe da APS - eAB/ eSF/eSB e eNASF. Município do estado de Goiás. 2020.

\begin{tabular}{|c|c|c|}
\hline \multirow{2}{*}{ EQUIPES } & PROFISSIONAIS & EJARCI \\
\hline & $\mathrm{N}(\%)$ & MÉDIA ( $\pm D P$ ) \\
\hline \multicolumn{3}{|l|}{ eAB/eSF/eSB } \\
\hline Agente Comunitário de Saúde & $23(25,3)$ & $114,74( \pm 11,27)$ \\
\hline Auxiliar Administrativo & $3(3,3)$ & $111,67( \pm 11,37)$ \\
\hline Enfermagem & $12(13,2)$ & $122,58( \pm 8,18)$ \\
\hline Medicina & $10(11,0)$ & $123,50( \pm 7,57)$ \\
\hline Odontologia & $8(8,8)$ & $123,57( \pm 5,85)$ \\
\hline Técnico ou auxiliar em Enfermagem & $17(18,7)$ & $119,71( \pm 11,36)$ \\
\hline Técnico ou auxiliar em Saúde Bucal & $4(4,4)$ & $117,75( \pm 16,37)$ \\
\hline \multicolumn{3}{|l|}{ eNASF } \\
\hline Educação física & $1(1,1)$ & $133^{*}$ \\
\hline Farmácia & $2(2,2)$ & $126,00( \pm 7,07)$ \\
\hline Fisioterapia & $4(4,4)$ & $125,75( \pm 4,78)$ \\
\hline Fonoaudiologia & $1(1,1)$ & $128^{*}$ \\
\hline Nutrição & $2(2,2)$ & $115,00( \pm 2,82)$ \\
\hline Psicologia & $2(2,2)$ & $133,00( \pm 2,83)$ \\
\hline Ignorados** & $2(2,2)$ & $112,5( \pm 31,8)$ \\
\hline
\end{tabular}

* Nota: valor absoluto ** Ignorados: profissionais que não informaram a categoria profissional

Fonte: Elaborada pelos autores.

atuação, percebem a importância do trabalho interprofissional entre a equipe nos serviços de saúde.

O fluxo de trabalho das eSF, eSB e eAB, incluindo os profissionais do administrativo foram observados. A principal diferença analisada entre as equipes foi a atuação da eAB com outros programas de saúde implementados na mesma estrutura física, como descrito no diário de campo:

Nesta UBS (com eAB), enfermeiro e médico generalista atuam de modo independente dos demais profissionais, inclusive em relação aos trabalhadores vinculados aos programas de tabagismo, tuberculose e hanseníase, e saúde da mulher. Já técnicos de enfermagem, farmacêutico e pessoal da recepção interagem como todos os profissionais, que são cinco enfermeiros e cinco médicos, dos quais dois são pediatras, dois ginecologistas e um dermatologista. (Diário de Campo. Dezembro, 2019).

As UBSs com eSF e eSB são situadas nas diferentes regiões do município e a capacidade de suas instalações físicas é bem diversificada. Contudo, todas possuem minimamente recepção, consultórios de atendimento médico, de enfermagem e odontológico, sala de vacina, sala de curativo, espaço para triagem, depósito de materiais, central de material e esterilização, banheiros e copa.

\section{Processo de trabalho em saúde - Fluxograma Analisador}

$\mathrm{Na}$ eAB, a rotina é bastante intensa e a lógica do trabalho ocorre da seguinte forma: os usuários chegam na recepção à procura de consulta, que são marcadas de acordo com a agenda do profissional, ou encaminhados via Sistema Nacional de Regulação (SISREG). Ao chegar, o usuário passa pela triagem, momento em que a técnica de enfermagem verifica os sinais vitais e o encaminha para aguardar atendimento médico ou de enfermagem.

Durante o período de coleta de dados observou-se que os profissionais trabalham de forma isolada, como poucos momentos de comunicação e troca de informações com vistas a continuidade do cuidado. Tais aspectos são reforçados pelo registro do diário de campo:

Na sala de recepção, em uma conversa informal com os usuários, um deles relatou que quando necessita de atendimento com profissionais de diferentes categorias, de fato esse atendimento ocorre e suas queixas são acolhidas. Porém, afirmou que não presenciou tais profissionais atuarem de forma articulada durante o seu atendimento. Foi possível observar a realização de encaminhamentos feitos por profissionais para colegas de outras equipes via ficha de referência e contrarreferência (Diário de Campo. Dezembro, 2019). 
Após a consulta, os usuários se direcionam a farmácia do local à procura do medicamento prescrito. Em seguida, a maioria passa na recepção para agendamento do próximo atendimento e/ou para sanar dúvidas. Esse fluxo de atendimento repetese no cotidiano da UBS, com pouca ou nenhuma diferença na prestação de cuidados ofertada pela $\mathrm{e} A \mathrm{~B}$ e de outros programas de saúde.

Segundo relatos dos trabalhadores, não há reuniões entre a equipe que compõe os programas. Entretanto, já presenciaram momentos em que houve discussão de casos entre o médico e a enfermeira, o que revela uma perspectiva interprofissional do trabalho em saúde. Aspecto reforçado pelo excerto do diário de campo:

Até o momento, não foi observado atendimento coletivo e as "pequenas reuniões" ou diálogos ocorrem de forma fragmentada entre as categorias nos corredores ou consultórios de forma pontual (Diário de Campo. Fevereiro, 2020).

Concernente às reuniões administrativas para discutir aspectos da organização do serviço, foi possível evidenciar que a coordenadora administrativa realiza reuniões pontuais na recepção na UBS. Além disso, observaram-se reuniões entre a equipe de enfermagem uma vez ao mês, liderada pela enfermeira coordenadora da equipe e os assuntos são relacionados à classe profissional. A Figura 1 expressa o fluxograma do processo de trabalho na UBS com eAB.

As UBS com eSF e eSB observadas possuem fluxos de trabalho semelhantes entre si. Em algumas unidades, a espera é organizada por senha com uso de painel digital, em outras os usuários chamados verbalmente no acolhimento pelos trabalhadores administrativos. Em todas as UBS, é verificado se seu atendimento é livre demanda ou agendamento.

Em ambos os casos, é realizada a triagem com verificação dos sinais vitais e medidas antropométricas. Os serviços prestados incluem pré-natal, puerpério, planejamento familiar, consulta ginecológica, consulta do homem, da criança, do adolescente, idoso, pessoas com doenças crônicas e consulta odontológica. De acordo com a necessidade, os usuários são encaminhados para suas respectivas consultas.

Hátambém oferta de procedimentos técnicos, como aferição de pressão arterial, glicemia e curativo por livre demanda. Isto é, passam pela recepção e são direcionados para equipe de enfermagem.

De modo geral, observamos que conversas entre os diferentes profissionais acontecem sobre casos em acompanhamento e são realizadas nos corredores entre uma consulta e outra, bem como nas reuniões informais durante o lanche da manhã na copa. $O$ trecho a seguir mostra tal aspecto:

Hoje pude participar de uma conversa entre os ACS, enfermeiros, dentistas, técnicos e médico. Estavam comentando sobreo grupo de idosos das quintas-feiras. Estavam discutindo como cada profissional poderia ajudar, pois a procura cresceu nos últimos meses (Diário de Campo. Janeiro, 2020).

Essas unidades oferecem diversas atividades coletivas, como o Programa de Saúde na Escola, com atuação em instituições de ensino municipais. Além disso, também são promovidos grupos como de gestantes e mistos que envolvem temáticas de

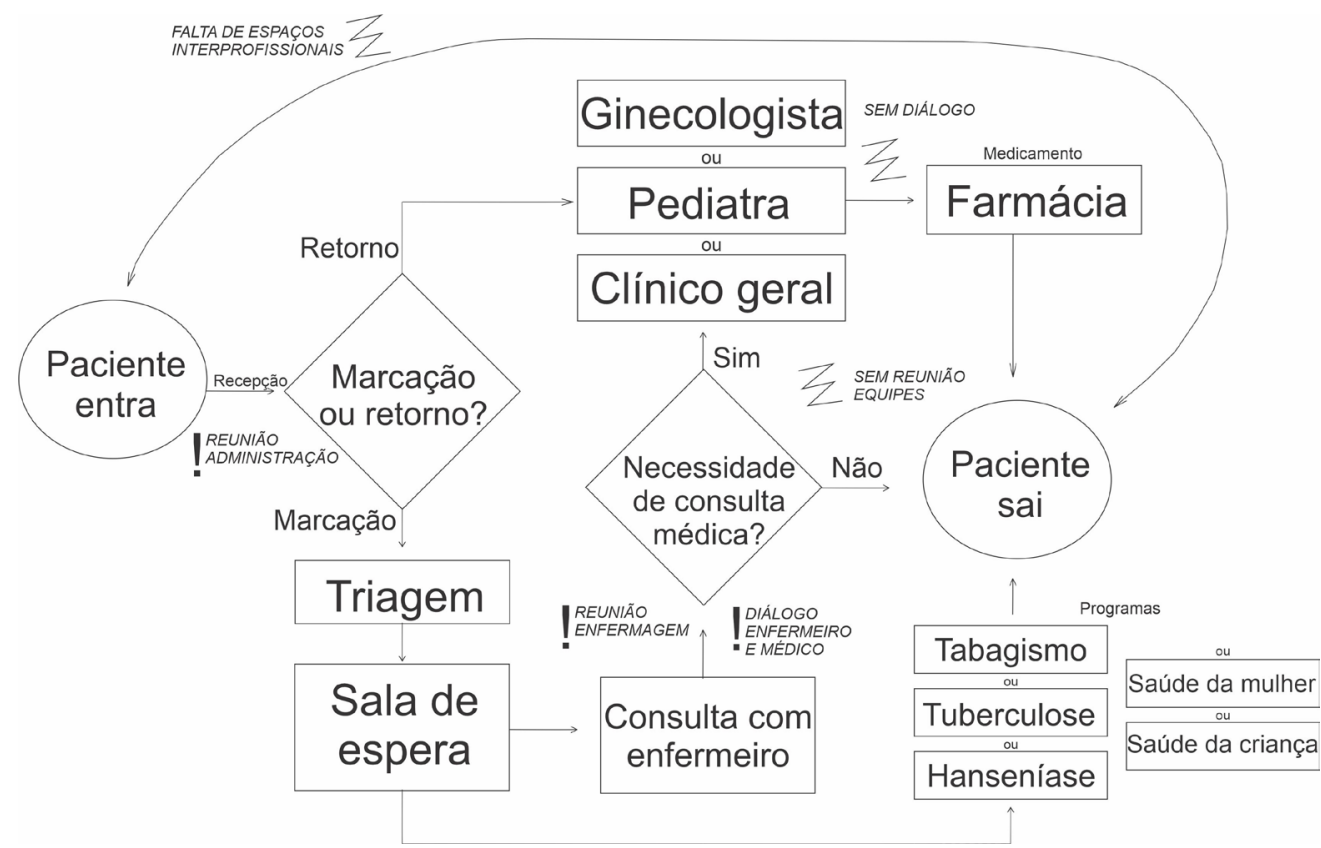

Figura 1 - Fluxograma do processo de trabalho centrado no usuário com enfoque na interprofissionalidade em UBS com eAB. Município do estado de Goiás. 2020.

Fonte: Elaborada pelos autores 
saúde e prevenção de agravos, bem como de artesanato nas unidades. Ocorre também educação em saúde coletiva na sala de espera da UBS ou no território, em igrejas, eventos, creches. O fluxograma do processo de trabalho consta na Figura 2.

\section{Atitudes colaborativas e interprofissionais entre os trabalhadores respondentes}

A Tabela 2 apresenta a frequência absoluta e relativa, mediana e moda das respostas aos 20 itens da escala EJARCI.

Os dados apresentados na tabela apontam fortes atitudes colaborativas e interprofissionais entre os trabalhadores que responderam aos questionários. Ao considerar-se tais resultados quantitativos e as observações do fluxo de atendimento dos serviços, onde os mesmos profissionais atuam, é possível evidenciar alguns aspectos divergentes.

As observações e os fluxos de atendimento das UBS analisadas mostram um processo de trabalho com diálogos e conversas entre os trabalhadores, pontuais e espontâneas. Contraditoriamente, $86,8 \%$ dos respondentes discordaram da afirmativa do item cinco da escala sobre os efeitos prejudiciais aos usuários causados por possíveis questionamentos entre a equipe de saúde. Na mesma direção, todos (100\%) os participantes concordaram que colegas com outras formações em saúde podem colaborar para qualidade do cuidado prestado (Item 13).

Ao tomarmos os fluxos de atendimento, tanto da $E A B$ quanto daquelas com eSF, observa-se um processo de trabalho estruturado por normas institucionais e protocolos próprios do serviço. Ao chegar na unidade, o usuário passa por processos decisórios prévios, por exemplo, se está agendado ou se busca por consultas ou procedimentos técnicos ou vacinação. Dessas decisões têm acesso ao cardápio de serviços prestados, já estipulados pelos diferentes programas de saúde e pela gestão.

Espaços para diálogos e interações interprofissionais podem ficar perdidos no fluxo unidirecional do atendimento. Apesar do processo de trabalho rígido, os trabalhadores participantes manifestaram no questionário EJARCI respostas opostas a essa rotina das UBS. $85,7 \%$ dos respondentes não estão de acordo com item 16 , cuja afirmativa se refere aos benefícios trazidos para os usuários das decisões e julgamentos isolados dos profissionais de saúde.

Congruentemente, $95.6 \%$ dos participantes confirmaram que todos os integrantes da equipe devem colaborar nas decisões relacionadas à melhora do cuidado das pessoas. Aspecto destoante da realidade abordada nesta pesquisa. Observamos diálogos rápidos em locais informais, como nos corredores e copas, sobre casos atendidos. Pontualmente, observamos o ACS relatar casos para os médicos. Apesar disso, todos (100\%) trabalhadores respondentes concordaram sobre a importância das relações de trabalho próximas para a prática colaborativa (Item 7). Essa afirmação pode refletir as conversas mais longas e acolhedoras observadas entre os profissionais durante o lanche na copa.

Como não foi observada agenda de reuniões nos serviços para discutir casos clínicos ou aspectos do trabalho, as decisões sobre o cuidado de cada indivíduo e sua família parece ser feita automaticamente. Mesmo nessa conjuntura, $92,3 \%$ dos respondentes da APS afirmaram que todos os profissionais podem contribuir nas tomadas de decisões para melhoria da assistência prestada (Item 11). E $85,8 \%$ concordaram sobre a participação da equipe na tomada de decisões político-administrativas em seu trabalho (Item 14).

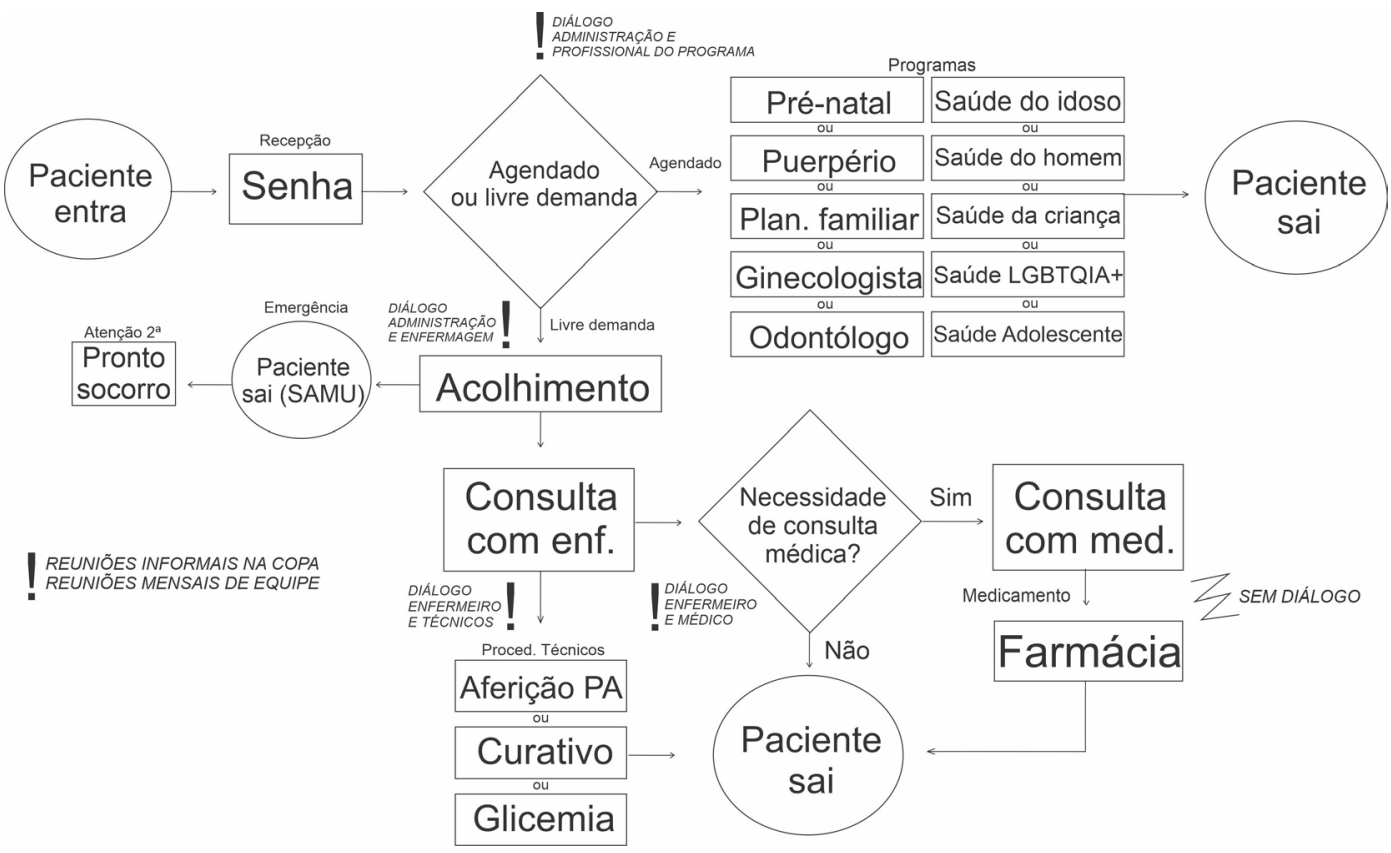

Figura 2 - Fluxograma do processo de trabalho centrado no usuário com enfoque na interprofissionalidade em UBS com eSF e eSB. Município do estado de Goiás. 2020.

Fonte: Elaborada pelos autores 
Tabela 2 - Síntese dos 20 itens da Escala Jefferson de Atitudes Relacionadas à Colaboração Interprofissional quanto à frequência absoluta e relativa, mediana e moda dos profissionais da Atenção Primária (n=91). Município do estado de Goiás. 2020.

\begin{tabular}{|c|c|c|c|c|c|c|}
\hline \multirow{2}{*}{ ITEM } & \multirow{2}{*}{ AFIRMATIVAS } & \multicolumn{5}{|c|}{ ESCALA* } \\
\hline & & $1-3$ & 4 & $5-7$ & Mediana & Moda \\
\hline 1 & $\begin{array}{l}\text { Os profissionais de saúde devem ser vistos como colaboradores, } \\
\text { em vez de superiores ou subordinados. }\end{array}$ & $3(3,3 \%)$ & $3(3,3 \%)$ & $85(93,4 \%)$ & 7 & 7 \\
\hline 2 & $\begin{array}{l}\text { Todos os profissionais de saúde devem ter a responsabilidade de } \\
\text { monitorar os efeitos de intervenções em seus pacientes/clientes. }\end{array}$ & $2(2,2 \%)$ & $6(6,6 \%)$ & $83(91,2 \%)$ & 7 & 7 \\
\hline 3 & $\begin{array}{l}\text { O trabalho em equipe no cuidado à saúde não pode ser um } \\
\text { resultado do ensino interdisciplinar. }\end{array}$ & $70(77 \%)$ & $7(7,7 \%)$ & $14(15,3 \%)$ & 2 & 1 \\
\hline 4 & $\begin{array}{l}\text { As instituições acadêmicas devem desenvolver programas de } \\
\text { ensino interdisciplinar para aumentar a prática colaborativa }\end{array}$ & $5(5,5 \%)$ & $6(6,6 \%)$ & $80(87,9 \%)$ & 7 & 7 \\
\hline 5 & $\begin{array}{l}\text { Os profissionais de saúde não devem questionar as decisões } \\
\text { tomadas por colegas, mesmo que eles achem que essas possam } \\
\text { ter efeitos prejudiciais ao paciente/cliente. }\end{array}$ & $79(86,8 \%)$ & $4(4,4 \%)$ & $8(8,8 \%)$ & 1 & 1 \\
\hline 6 & $\begin{array}{l}\text { Todos os profissionais de saúde podem contribuir para as } \\
\text { decisões relacionadas ao bem-estar de pacientes/clientes. }\end{array}$ & $5(5,5 \%)$ & $2(2,2 \%)$ & $84(92,3 \%)$ & 7 & 7 \\
\hline 7 & $\begin{array}{l}\text { A prática colaborativa sempre funciona melhor quando os } \\
\text { profissionais de saúde desenvolvem relações de trabalho para } \\
\text { atingir os mesmos objetivos. }\end{array}$ & $0(0,0 \%)$ & $0(0,0 \%)$ & $91(100 \%)$ & 7 & 7 \\
\hline 8 & $\begin{array}{l}\text { O ensino interdisciplinar e a colaboração interprofissional não } \\
\text { estão ligados entre si. }\end{array}$ & $69(75,8 \%)$ & $10(11,0 \%)$ & $12(13,2 \%)$ & 1 & 1 \\
\hline 9 & $\begin{array}{l}\text { A função principal de outros profissionais de saúde é seguir, } \\
\text { sem questionamento, as orientações dos médicos que estão } \\
\text { tratando os pacientes/clientes. }\end{array}$ & $65(71,5 \%)$ & $8(8,8 \%)$ & $18(19,7 \%)$ & 2 & 1 \\
\hline 10 & $\begin{array}{l}\text { A colaboração interprofissional, que inclui respeito mútuo e } \\
\text { comunicação, melhora o ambiente de trabalho. }\end{array}$ & $0(0,0 \%)$ & $0(0,0 \%)$ & $91(100 \%)$ & 7 & 7 \\
\hline 11 & $\begin{array}{l}\text { Todos os profissionais de saúde devem contribuir para as decisões } \\
\text { relativas à melhora do cuidado de seus pacientes/clientes. }\end{array}$ & $1(1,1 \%)$ & $0(0,0 \%)$ & $90(98,9 \%)$ & 7 & 7 \\
\hline 13 & $\begin{array}{l}\text { Os profissionais de saúde devem estar cientes de que seus } \\
\text { colegas de outras áreas relacionadas à saúde podem contribuir } \\
\text { para a qualidade do cuidado. }\end{array}$ & $0(0,0 \%)$ & $0(0,0 \%)$ & $91(100 \%)$ & 7 & 7 \\
\hline 14 & $\begin{array}{l}\text { Os profissionais de saúde devem estar envolvidos na tomada de } \\
\text { decisões político-administrativas relativas ao seu trabalho. }\end{array}$ & $5(5,5 \%)$ & $8(8,8 \%)$ & $78(85,8 \%)$ & 6 & 7 \\
\hline 15 & $\begin{array}{l}\text { Devido à diferença de cada função, não há muitas áreas que } \\
\text { permitam a sobreposição de responsabilidades entre os profissionais } \\
\text { de saúde que prestam cuidados aos pacientes/clientes. }\end{array}$ & $32(35,1 \%)$ & $23(25,3 \%)$ & $36(39,6 \%)$ & 4 & 4 \\
\hline 16 & $\begin{array}{l}\text { Para promover o melhor benefício ao paciente/cliente, os } \\
\text { profissionais de saúde devem usar seu próprio julgamento em vez } \\
\text { de consultar seus colegas de outras áreas relacionadas à saúde. }\end{array}$ & $78(85,7 \%)$ & $5(5,5 \%)$ & $8(8,8 \%)$ & 1 & 1 \\
\hline 17 & $\begin{array}{l}\text { Os erros clínicos serão minimizados quando existir colaboração } \\
\text { entre os profissionais de saúde. }\end{array}$ & $8(8,8 \%)$ & $5(5,5 \%)$ & $78(85,7 \%)$ & 7 & 7 \\
\hline 18 & $\begin{array}{l}\text { Todos os profissionais de saúde possuem competências } \\
\text { específicas próprias para prestar atendimento de qualidade aos } \\
\text { seus pacientes / clientes. }\end{array}$ & $4(4,4 \%)$ & $7(7,6 \%)$ & $80(88 \%)$ & 6 & 7 \\
\hline 19 & $\begin{array}{l}\text { Os profissionais de saúde que trabalham em conjunto não podem } \\
\text { ser igualmente responsabilizados pelo serviço que prestam. }\end{array}$ & $54(59,3 \%)$ & $9(9,9 \%)$ & $28(30,8 \%)$ & 3 & 1 \\
\hline 20 & $\begin{array}{c}\text { Durante sua formação, todos os estudantes da área da } \\
\text { saúde devem ter a experiência de trabalhar em equipes } \\
\text { com estudantes de outras áreas da saúde, para que possam } \\
\text { compreender melhor sua respectiva função. }\end{array}$ & $6(6,6 \%)$ & $0(0,0 \%)$ & $85(93,4 \%)$ & 7 & 7 \\
\hline
\end{tabular}

* Nota: Escala 1 (discordo completamente) a 7 (concordo completamente)

Fonte: Elaborada pelos autores 


\section{DISCUSSÃO}

Os resultados evidenciaram incongruência entre a realidade observada e as questões respondidas pelos trabalhadores. Os processos de trabalho das UBS estudadas produzem um agir em saúde pouco colaborativo entre as categorias profissionais. Em contrapartida, as respostas à escala de avaliação da interprofissionalidade apontaram fortes atitudes colaborativas e interprofissionais, independentemente da configuração da equipe.

Apesar das condições de trabalho e área de atuação, os dados apontaram que os profissionais percebem a importância do trabalho colaborativo e das relações interprofissionais nos serviços de saúde. Esse aspecto sinaliza que os discretos indícios observados do trabalho colaborativo não implicam hostilidade e competição, nem excluem o desejo do trabalho colaborativo entre eles. Há uma pluralidade de fatores, desde individuais, relações interpessoais, até condições organizacionais, que influenciam a atuação interprofissional nos cuidados em saúde ${ }^{18}$.

Em relação às condições materiais, as observações deste estudo, ao passo que, evidenciaram poucas unidades com salas próprias para reunião, mostraram baixo índice dessa atividade entre os profissionais. A infraestrutura que não favorece uma prática interprofissional pode ser características de muitas UBS. A qualidade estrutural é deficiente em todo o Brasil, com apenas $4,8 \%$ dos serviços classificadas como "A", aquelas que contemplam todo o conjunto de critérios do padrão de referência, inclusive espaço próprio para encontros coletivos ${ }^{19}$.

Estudos apontam que reuniões programadas são elencadas como uma das estratégias para a consolidação da prática interprofissional ${ }^{20-21}$ A comunicação atua como um suporte para o trabalho colaborativo ${ }^{14}$. De modo semelhante, outros estudos apontam pouca ou nenhuma ocorrência de reuniões nos serviços para discussão de casos clínicos ou aspectos da organização do serviço ${ }^{14,21}$.

O diálogo entre os profissionais de saúde é uma ação inerente ao trabalho em equipe e à tomada de decisão de forma democrática ${ }^{20}$. Apesar de os profissionais reconhecerem a importância da comunicação ao responderem a EJARCI, observamos conversas pontuais e informais no processo de trabalho. A fim de compreender tal contradição, vale destacar que o tema da interprofissionalidade é relativamente novo no contexto da APS do Brasil, aspecto reiterado pelo número discreto de manuscritos na literatura brasileira, com publicação predominante nos últimos cinco anos ${ }^{4,22}$.

Considerando que parcela importante dos profissionais da APS possui tempo de formação e tempo de atuação no SUS superior a uma década ${ }^{23-24}$, inclusive os participantes deste estudo, é imprescindível reforçar a educação interprofissional.

Esse tipo de educação permanente favorece o alinhamento do trabalho em saúde ao cuidado integral, para uma visão além das formações acadêmicas básicas, pautadas por um modelo de saúde assistencialista e fragmentado ${ }^{23}$. Modelo que não comporta as mudanças necessárias para a prestação integral de cuidados, prática interprofissional e trabalho colaborativo, aspectos ansiados na atenção primária ${ }^{24}$.
As respostas divergentes em relação ao contexto observado também podem sinalizar que os profissionais participantes possuem motivação para desenvolver práticas colaborativas. Todavia, como discutido, a organização do trabalho parece não as favorecer. Estudo sobre a força motivacional dos profissionais da APS na região Nordeste do país identificou eventos processuais como causas do declínio paulatino da motivação no ambiente de trabalho. Tais quais, condições de trabalho desfavoráveis, falta de capacitação e falta de estrutura. Aponta, ainda, que o fator motivacional pode se alterar ao longo do tempo, nisto, práticas colaborativas podem ser uma estratégia motivacional e de otimização da atenção em saúde ${ }^{25}$.

Nesse contexto, salienta-se a potencialidade da educação interprofissional, cuja propostaé envolver os estudantes, trabalhadores, usuários e familiares na atenção em saúde, de forma colaborativa. No cenário estudado, é uma importante estratégia de valorização das intencionalidades verificadas dos profissionais para o trabalho colaborativo, visto que se configura como uma das formas mais promissoras para o fortalecimento da colaboração da equipe de trabalho ${ }^{26}$. Também pode ser a maneira mais resolutiva para o desenvolvimento da interprofissionalidade em equipes profissionais que reconhece sua importância, porém não a pratica.

\section{CONSIDERAÇÕES FINAIS E IMPLICAÇÕES PARA A PRÁTICA}

Há divergências entre o falado e o vivido em relação à interprofissionalidade na rotina de trabalho nos serviços de APS estudados. Apesar de os trabalhadores relatarem fortes atitudes colaborativas, os processos de trabalho observados evidenciaram poucas situações de práticas interprofissionais.

A média para atitudes colaborativas obtida a partir das respostas ao questionário EJARCI foi alta. Todavia, a organização do trabalho de forma rígida limita a interprofissionalidade no cuidado na atenção primária. Ao se comparar o trabalho observado nas UBS com eSF e eSB com a UBS com eAB, as primeiras apresentaram mais espaços e oportunidades convergentes para o trabalho colaborativo.

Como limitações deste estudo, é válido mencionar a não inclusão da eNASF na fase qualitativa. Na etapa quantitativa, não foi possível estabelecer correlações. Apesar disso, a metodologia empregada neste estudo permitiu evidenciar, independente do tipo de equipe na atenção primária, se eSF ou eSB ou eAB, que o processo de trabalho estruturado e vivido por esses trabalhadores parece enfraquecer possíveis atitudes interprofissionais.

A partir dos achados apontamos a necessidade de mais investigações com abordagem de métodos mistos sobre a colaboração interprofissional. É importante incluir amostras populacionais representativas, a fim de estabelecer correlações. E a partir delas, compreender em profundidade como o contexto de trabalho e as políticas públicas impactam o trabalho em equipe na atenção primária, especialmente, durante e a partir da atuação profissional na pandemia do Sars-Cov-2.

Salientamos como implicações para a prática assistencial a valorização de atividades programadas para o trabalho interprofissional da equipe de saúde e de enfermagem na atenção 
primária, tais como, espaços na agenda para reuniões com objetivo de discutir casos e a organização do trabalho. Outro aspecto fundamental é a realização da educação interprofissional com os trabalhadores, para que, coletivamente, possam encontrar estratégias transformadoras da dinâmica de trabalho na APS e caminhos para concretizar suas motivações para o agir e cuidar colaborativos. Nesse contexto, torna-se essencial que as políticas públicas da atenção básica, mais que apontar configurações, atribuições e horários de atendimento, possam garantir mecanismos para processos de trabalho interprofissionais, valorizadores das intencionalidades das equipes e centrados no usuário.

\section{CONTRIBUIÇÕES DOS AUTORES}

Desenho do estudo de revisão. Aridiane Alves Ribeiro. Christiane Ricaldoni Giviziez. Elânia Assis Rocha Coimbra. Jeniffer Dayane Duarte dos Santos. Jhonatan Emanuel Maciel de Pontes. Nina Franco Luz. Rafael de Oliveira Rocha. Webster Leonardo Guimarães da Costa

Aquisição dos textos. Aridiane Alves Ribeiro. Christiane Ricaldoni Giviziez. Elânia Assis Rocha Coimbra. Jeniffer Dayane Duarte dos Santos. Jhonatan Emanuel Maciel de Pontes. Nina Franco Luz. Rafael de Oliveira Rocha. Webster Leonardo Guimarães da Costa

Análise de dados. Aridiane Alves Ribeiro. Christiane Ricaldoni Giviziez. Elânia Assis Rocha Coimbra. Jeniffer Dayane Duarte dos Santos. Jhonatan Emanuel Maciel de Pontes. Nina Franco Luz. Rafael de Oliveira Rocha. Webster Leonardo Guimarães da Costa

Interpretação dos resultados. Aridiane Alves Ribeiro. Christiane Ricaldoni Giviziez. Elânia Assis Rocha Coimbra. Jeniffer Dayane Duarte dos Santos. Jhonatan Emanuel Maciel de Pontes. Nina Franco Luz. Rafael de Oliveira Rocha. Webster Leonardo Guimarães da Costa

Redação e revisão crítica do manuscrito. Aridiane Alves Ribeiro. Christiane Ricaldoni Giviziez. Elânia Assis Rocha Coimbra. Jeniffer Dayane Duarte dos Santos. Jhonatan Emanuel Maciel de Pontes. Nina Franco Luz. Rafael de Oliveira Rocha. Webster Leonardo Guimarães da Costa

Aprovação da versão final do artigo. Aridiane Alves Ribeiro. Christiane Ricaldoni Giviziez. Elânia Assis Rocha Coimbra. Jeniffer Dayane Duarte dos Santos. Jhonatan Emanuel Maciel de Pontes. Nina Franco Luz. Rafael de Oliveira Rocha. Webster Leonardo Guimarães da Costa

Responsabilidade por todos os aspectos do conteúdo e a integridade do artigo publicado Aridiane Alves Ribeiro. Christiane Ricaldoni Giviziez. Elânia Assis Rocha Coimbra. Jeniffer Dayane Duarte dos Santos. Jhonatan Emanuel Maciel de Pontes. Nina Franco Luz. Rafael de Oliveira Rocha. Webster Leonardo Guimarães da Costa

\section{EDITOR CIENTÍFICO}

Ivone Evangelista Cabral (1)

\section{EDITOR ASSOCIADO}

Beatriz Rosana Gonçalves de Oliveira Toso (1)

\section{REFERÊNCIAS}

1. Samelli AG, Tomazelli GA, Almeida MHM, Oliver FC, Melo SR, Avejonas DRM. Avaliação da atenção ao bebê de risco: comparação entre modelos de atenção primária à saúde. Rev Saude Publica. 2019;18(53) PMid:31778397.

2. Geremia DS. Atenção primária à saúde em alerta: desafios da continuidade do modelo assistencial. Physis. 2020;30(1):e300100. http://dx.doi.org/10.1590/s0103-73312020300100.

3. Silva JAM, Peduzzi M, Orchard C, Leonello VM. Educação interprofissiona e prática colaborativa na Atenção Primária à Saúde. Rev Esc Enferm USP. 2015;49(spe2):16-24. http://dx.doi.org/10.1590/S0080623420150000800003

4. Farias DN, Ribeiro KSQS, Anjos UU, Brito GEG. Interdisciplinaridade e Interprofissionalidade na Estratégia Saúde da Família. Trab Educ Saúde. 2017;16(1):141-62. http://dx.doi.org/10.1590/1981-7746-sol00098.

5. Reeves S, Xyrichis A, Zwarenstein M. Teamwork, collaboration, coordination, and networking: Why we need to distinguish between different types of interprofessional practice. J Interprof Care. 2018;32(1):1-3. http:// dx.doi.org/10.1080/13561820.2017.1400150. PMid:29131697.

6. Oliveira AV, Sabino BCN, Dias LR, Gouvea ABV, Garbelini GU, Paiva IG. Avaliação da longitudinalidade em unidades básicas de saúde tradicionais e com estratégia de saúde da família utilizando o Primary Care Assessment Tool (PCATool). Revista Eletrônica Acervo Saúde. 2020;12(12):e3895.

7. Portaria $\mathrm{n}^{\circ} \mathbf{2} .436$ de 21 de setembro de 2017 (BR). Aprova a Política Nacional de Atenção Básica, estabelecendo a revisão de diretrizes para a organização da Atenção Básica, no âmbito do Sistema Único de Saúde (SUS). Diário Oficial da União, Brasília (DF), 21 set 2017.

8. Giovanella L. Atenção básica ou atenção primária à saúde? Cad Saude Publica. 2018;34(8):e00029818. http://dx.doi.org/10.1590/0102311x00029818. PMid:30133652.

9. Merhy EE, Chakkour M, Stéfano E, Stéfano ME, Santos CM, Rodrigues RA et al. Em busca de ferramentas analisadoras das tecnologias em saúde: a informação e o dia a dia de um serviço, interrogando e gerindo trabalho em saúde. In: Merhy EE, Onocko R, organizadores. Agir em saúde: um desafio para o público. São Paulo: Hucitec;2007. p. 113-50.

10. Vila VSC, Rossi LA. O significado cultural do cuidado humanizado em unidade de terapia intensiva: "muito falado e pouco vivido". Rev Lat Am Enfermagem. 2002;10(2):137-44. http://dx.doi.org/10.1590/ S0104-11692002000200003.

11. Minayo MCS, Deslandes SF, Gomes R. Pesquisa social: teoria, método e criatividade. Petrópolis: Vozes; 2013.

12. Secretaria de Saúde do Estado de Goiás. Regionais de Saúde [Internet] Goiânia: Secretaria de Saúde do Estado de Goiás; 2020 [citado 18 set 2020]. Disponível em: https://www.saude.go.gov.br/unidades-de-saude/ regioes-de-saude

13. Brasil. Ministério da Saúde. Cobertura-AB-CENTRO-OESTE - GO JATAI'-Janeiro de 2020 [Internet]. Brasília: e-Gestor Atenção Básica; 2020 [citado 18 set 2020]. Disponível em: https://egestorab.saude.gov. br/paginas/acessoPublico/relatorios/relHistoricoCoberturaAB.xhtml

14. Canadian Interprofessional Health Collaborative College of Health Disciplines. A 20. National Interprofessional Competency Framework. Vancouver: CIHC; 2010.

15. Bardin, L. Análise de conteúdo. São Paulo: Almedina; 2016.

16. Hojat M, Ward J, Spandorfer J, Arenson C, Van Winkle LJ, Williams B (JeffSATIC): development and multi-institution psychometric data. J Interprof Care. 2015;29(3):238-44. http://dx.doi.org/10.3109/135618 20.2014.962129. PMid:25311270.

17. Abed MM. Adaptação e validação da versão brasileira da Escala Jefferson de Atitudes Relacionadas à Colaboração Interprofissional: um estudo em profissionais da atenção básica [dissertação]. Goiânia: Universidade Federal de Goiás; 2015.

18. van Dongen JJJ, Lenzen SA, van Bokhoven MA, Daniëls R, van de Weijden T, Beurskens A. Interprofessional collaboration regarding patients' care plans in primary care: a focus group study into influential factors. BMC Fam Pract. 2016;17(1):58. http://dx.doi.org/10.1186/ s12875-016-0456-5. PMid:27233362. 
19. Bousquat A, Giovanella L, Fausto MCR, Fusaro ER, Mendonça MHM, Gagno J et al. Tipologia da estrutura das unidades básicas de saúde brasileiras: os 5 R. Cad Saude Publica. 2017;33(8):e00037316. http:// dx.doi.org/10.1590/0102-311x00037316. PMid:28832772.

20. Reuter CLO, Santos VCF, Ramos AR. The exercise of interprofessionality and intersetoriality as an art of caring: innovations and challenges. Esc Anna Nery.2018;22(4). http://dx.doi.org/10.1590/2177-9465-ean-2017-0441.

21. Santos PF, Pedrosa KP, Pinto JR. A educação permanente como ferramenta no trabalho interprofissional na Atenção Primária à Saúde. Tempus Actas de Saúde Coletiva. 2016;10(3):177. http://dx.doi. org/10.18569/tempus.v10i3.1641.

22. Mallmann FH, Toasse RFC. Educação e trabalho interprofissional em saúde no context da atenção primária no Brasil: análise da produção científica de 2010 a 2017. Sab Plur. 2019;3(1):70-84.
23. Barros NF, Spadacio C, Costa MV. Trabalho interprofissional e as práticas integrativas e complementares no contexto da Atenção Primária à Saúde: potenciais e desafios. Saúde Debate. 2018;42(spe1):163-73. http://dx.doi.org/10.1590/0103-11042018s111.

24. Fowler T, Garr D, Mager ND, Stanley J. Enhancing primary care and preventive services through Interprofessional practice and education. Isr J Health Policy Res. 2020;9(1):12. http://dx.doi.org/10.1186/s13584020-00371-8. PMid:32204734.

25. Alves A Fo, Borges LO. A motivação dos profissionais de saúde das Unidades Básicas de Saúde. Psicologia. 2014;34(4):984-1001. http:// dx.doi.org/10.1590/1982-370001082013.

26. Caneppele AH, Cucolo DF, Mininel VA, Meireles E, Silva JAM. Interprofessional collaboration in urgency and emergency network teams in the Covid-19 pandemic. Escola Anna Nery. 2020;24(spe). 